Pacific

Journal of

Mathematics

THE MAXIMUM PRINCIPLE FOR SYSTEMS OF PARABOLIC EQUATIONS SUBJECT TO AN AVOIDANCE SET

Bennett Chow and Peng Lu

Volume $214 \quad$ No. 2

April 2004 


\title{
THE MAXIMUM PRINCIPLE FOR SYSTEMS OF PARABOLIC EQUATIONS SUBJECT TO AN AVOIDANCE SET
}

\author{
Bennett Chow and Peng Lu
}

\begin{abstract}
Hamilton's maximum principle for systems states that given a reaction-diffusion equation (semi-linear heat-type equation) for sections of a vector bundle over a manifold, if the solution is initially in a subset invariant under parallel translation and convex in the fibers and if the ODE associated to the PDE preserves the subset, then the solution remains in the subset for positive time. We generalize this result to the case where the subsets are time-dependent and where there is an avoidance set from which the solution is disjoint. In applications the existence of an avoidance set can sometimes be used to prove the preservation of a subset of the vector bundle by the PDE.
\end{abstract}

\section{Introduction.}

For scalar parabolic equations, maximum principles are well-known [PW] and have been applied in numerous settings in partial differential equations and geometric analysis. In the case of systems of parabolic equations, maximum principles are not as well-known and appear to be much less frequent. Notable exceptions are given by Richard Hamilton $([\mathbf{H} \mathbf{1}]$ and [H2]) and Joel Smoller [S] (see Chapter 14). ${ }^{1}$ Hamilton's maximum principle holds for solutions of reaction-diffusion equations (PDE) which are time-dependent sections of a vector bundle over a Riemannian manifold; in particular, it holds for the reaction-diffusion equations satisfied by the curvature operator under Ricci flow. When the convex subsets of the fibers are independent of time, Hamilton proved such a maximum principle in [H2], which roughly says that if the convex subsets are preserved by the system of ordinary differential equations (ODE) associated to the PDE then the convex subsets are preserved by the PDE. A special case of this result, which applied to symmetric 2-tensors, was proved earlier by Hamilton in [H1] and applied to obtain crucial curvature pinching estimates in his proof that a compact 3-manifold with positive Ricci curvature converges to a constant curvature metric under the volume preserving Ricci flow. The general formulation in

\footnotetext{
${ }^{1}$ We would like to thank Yung-Sze Choi for informing us of this reference.
} 
[H2] greatly simplified the computations in [H1] and facilitated the more complicated convex analysis of the ODE associated to the evolution of the Riemann curvature operator in dimension four in [H5].

The main purpose of this paper is to prove two extensions of Hamilton's maximum principle for systems which should be useful for the study of the Ricci flow and some other geometric evolution equations such as the mean curvature flow. We present an extension where the convex sets are allowed to depend on time, we call the extension the maximum principle with timedependent convex sets (see Theorem 3). We also present a souped-up version where both the convex sets are allowed to depend on time and the convex set may not be preserved by the ODE on a subset of the boundary but the solution to the PDE avoids that part of the boundary. We call such subsets of the convex sets, which contain this part of their boundary, avoidance sets, and call this extension the maximum principle subject to an avoidance set (see Theorem 4).

A special case of this maximum principle with time-dependent convex sets has already been proved by Hamilton in Theorem 3.3 in Section 2.3 of [H5]. In particular, Hamilton adjoins to the solution $\sigma$ of the PDE the function $r=\frac{1}{T_{*}-t}$, where $T_{*}$ is the singularity time, which trivially satisfies the equation $\frac{\partial r}{\partial t}=\Delta r+r^{2}$ and applies the maximum principle to the pair $(\sigma, r)$. However, in general this device of adjoining the function $r=\frac{1}{T-t}$ has the drawback that the sets in space and time to be preserved may not be convex even though the space slices are. In such a case Hamilton's proof would not directly apply. In the proof of the somewhat more general form of the maximum principle given in this paper, we modify Hamilton's original proof of the maximum principle in [H2]. The difficulty in this approach is reconciling the time-dependence of the sets over which one takes the maximum of certain functions with the framework of Hamilton's "ODE to PDE" formulation. More precisely, when the convex sets depend on time, the lemma used by Hamilton (see Lemma 9) on taking the time derivative of the function $\sup _{s \in \mathcal{S}(t)} g(s, t)$ must have a correction term, since now the set $\mathcal{S}(t)$ depends on time, which is difficult to control in the later applications of the lemma. This is overcome by considering the space-time track of the timedependent convex sets and finding suitable splitting of certain quantities which arise in the study of both the ODE and the PDE (see the proof of Proposition 10 and the proof of Theorem 3). Formulating the proof this way enables us to generalize our result to the case where the PDE is subject to an avoidance set without much difficulty. A special case of this maximum principle has already been applied in $[\mathbf{H 5}]$ and $[\mathbf{H 6}]$ to obtain refined and subtle pointwise curvature estimates. In addition, although it is not necessary, our maximum principle may be used in the proofs of results in $[\mathbf{H 3}]$ and $[\mathbf{H 4}]$. 
Our maximum principle subject to avoidance sets is a more general formulation of a form of the maximum principle implicitly used in the proof of certain estimates in Section 2.3 of [H5] which are used to detect necks. Some of these estimates, have analogues in dimension three (see $\S 24$ of [H4]). In regards to this, as suggested by Mao-Pei Tsui, our souped-up version can also be used to give an alternate proof of Theorem 24.6 of [H4] (cf. [CK]). However, in the study of 4-manifolds with positive isotropic curvature [H5], a souped-up version is necessary and is implicitly used by Hamilton.

\section{Main results.}

Let $M^{n}$ be a closed oriented $n$-dimensional manifold with a smooth family of Riemannian metrics $g(t), t \in[0, T]$. Let $V \rightarrow M$ be a real vector bundle with a time-independent bundle metric $h$ and $\Gamma(V)$ be the vector space of $C^{\infty}$ sections of $V$. Let

$$
\nabla(t): \Gamma(V) \rightarrow \Gamma\left(V \otimes T M^{*}\right), t \in[0, T]
$$

be a smooth family of time-dependent connections compatible with $h$, that is,

$$
X[h(\sigma, \tau)]=h\left(\nabla(t)_{X} \sigma, \tau\right)+h\left(\sigma, \nabla(t)_{X} \tau\right)
$$

for all $X \in T M, \sigma, \tau \in \Gamma(V)$ and $t \in[0, T]$. The time-dependent Laplacian $\Delta(t)$ acting on a section $\sigma \in \Gamma(V)$ is defined by

$$
\Delta(t) \sigma=\operatorname{trace}_{g(t)}(\widehat{\nabla}(t)(\nabla(t) \sigma)),
$$

where

$$
\widehat{\nabla}(t): \Gamma\left(V \otimes T M^{*}\right) \rightarrow \Gamma\left(V \otimes T M^{*} \otimes T M^{*}\right)
$$

is defined using the connection $\nabla(t)$ on $V$ and the Levi-Civita connection $D(t)$ on $T M^{*}$ associated with metric $g(t)$. That is,

$$
\widehat{\nabla}(t)_{X}(\sigma \otimes \alpha)=\left(\nabla(t)_{X} \sigma\right) \otimes \alpha+\sigma \otimes\left(D(t)_{X} \alpha\right)
$$

for all $X \in T M, \sigma \in \Gamma(V), \alpha \in \Gamma\left(T M^{*}\right)$.

Let $F: V \times[0, T] \rightarrow V$ be a fiber preserving map; i.e., $F(\sigma, t)$ is a timedependent vector field defined on the bundle $V$ and tangent to the fibers. Then we can form a system of reaction-diffusion equations (PDE)

$$
\frac{\partial}{\partial t} \sigma(x, t)=\Delta(t) \sigma(x, t)+F(\sigma(x, t), t),
$$

where $\sigma(\cdot, t), t \in[0, T]$ are sections of $V$. In each fiber $V_{x}$ the system of ordinary differential equations (ODE) associated to the PDE (1) obtained by dropping the Laplacian term is

$$
\frac{d}{d t} \sigma_{x}(t)=F\left(\sigma_{x}(t), t\right)
$$

where $\sigma_{x}(t) \in V_{x}$. 
Let $\mathcal{K}$ be closed subset of $V$. Denote $\mathcal{K}_{x} \doteqdot \mathcal{K} \cap V_{x}$. For any initial time $t_{0} \in[0, T)$ we say that the solution $\sigma(x, t): t \in\left[t_{0}, T\right]$ of the PDE (1) starts in $\mathcal{K}$ if $\sigma\left(x, t_{0}\right) \in \mathcal{K}_{x}$ for all $x \in M$. We say that the solution $\sigma(x, t)$ remains in $\mathcal{K}$ for all later times if $\sigma(x, t) \in \mathcal{K}_{x}$ for all $x \in M$ and all $t \in\left(t_{0}, T\right]$. For any $x \in M$ and for any initial time $t_{0} \in[0, T)$ we say that the solution $\sigma_{x}(t): t \in\left[t_{0}, T\right]$ of the $\operatorname{ODE}(2)$ starts in $\mathcal{K}_{x}$ if $\sigma_{x}\left(t_{0}\right) \in \mathcal{K}_{x}$. We say that the solution $\sigma_{x}(t)$ remains in $\mathcal{K}_{x}$ for all later times if $\sigma_{x}(t) \in \mathcal{K}_{x}$ for all $t \in\left(t_{0}, T\right]$.

One important question is: When will an arbitrary solution of the PDE (1) which starts in $\mathcal{K}$ at an arbitrary initial time $t_{0} \in[0, T)$ remain in $\mathcal{K}$ for all later times? To answer this question, we need to impose two conditions on $\mathcal{K}$ :

I. $\mathcal{K}$ is invariant under parallel translation defined by the connection $\nabla(t)$ for each $t \in[0, T]$.

II. In each fiber $V_{x}$ set $\mathcal{K}_{x}$ is closed and convex.

The following theorem is the maximum principle of Hamilton (Theorem 4.3 in $[\mathbf{H 2}])$ :

Theorem 2. Let $\mathcal{K} \subset V$ be a closed subset satisfying Conditions I and II. Assume that $F(\sigma, t)$ is continuous in $t$ and is Lipschitz in $\sigma$. Suppose that for any $x \in M$ and any initial time $t_{0} \in[0, T)$, and any solution $\sigma_{x}(t)$ of the $\mathrm{ODE}(2)$ which starts in $\mathcal{K}_{x}$ at $t_{0}$, the solution $\sigma_{x}(t)$ will remain in $\mathcal{K}_{x}$ for all later times. Then for any initial time $t_{0} \in[0, T)$ the solution $\sigma(x, t)$ of the PDE (1) will remain in $\mathcal{K}$ for all later times if $\sigma(x, t)$ starts in $\mathcal{K}$ at time $t_{0}$.

In applications to the Ricci flow the vector bundle $V$ is a tensor bundle and the subsets $\mathcal{K}_{x} \subset V_{x}$, which are invariant under the action of $O(n)$, are identified under the isomorphism between two fibers $V_{x}$ and $V_{y}$ induced by any choice of orthonormal frames in $T M$ at the two points $x$ and $y$. The ODEs in $V_{x}$ and $V_{y}$ are also $O(n)$-invariant and identical under this identification. When this is the case the requirement on the solutions of the ODE (2) in Theorem 2 will hold for every fiber if it holds for one fiber.

Next we formulate the maximum principle where the convex sets are timedependent. Let $U$ be an open subset of $V$ and $\mathcal{K}(t) \subset U$ be a closed subset for each $t \in[0, T]$. We impose two conditions on $\mathcal{K}(t)$ for each $t$ :

III. $\mathcal{K}(t)$ is invariant under parallel translation defined by the connection $\nabla(t)$ for each $t \in[0, T]$.

IV. In each fiber $V_{x}$ set $\mathcal{K}_{x}(t) \doteqdot \mathcal{K}(t) \cap V_{x}$ is nonempty, closed and convex for each $t \in[0, T]$.

We define the space-time track

$$
\mathcal{T} \doteqdot\{(v, t) \in V \times[0, T]: v \in \mathcal{K}(t), t \in[0, T]\}
$$


and define

$$
\mathcal{T}_{x} \doteqdot \mathcal{T} \cap\left(V_{x} \times[0, T]\right)
$$

Let $F: U \times[0, T] \rightarrow V$ be a fiber preserving map, i.e., $F(x, \sigma, t)$ is a time-dependent vector field defined on $U$ and tangent to the fibers. Let $u(x, t): V_{x} \times T_{x} M^{*} \rightarrow V_{x}$ be a smooth family of bundle maps of diagonal form, i.e.,

$$
u(x, t)\left(\sigma, d x^{i}\right)=u^{i}(x, t) \cdot \sigma
$$

where $u^{i}(x, t)$ are smooth functions (in applications to the Ricci flow, $u \equiv 0$; that is, there is no gradient term). Then we can form a system of reactiondiffusion equations (PDE)

$$
\frac{\partial}{\partial t} \sigma(x, t)=\Delta(t) \sigma(x, t)+u(x, t)(\nabla(t) \sigma(x, t))+F(x, \sigma(x, t), t) .
$$

In each fiber $V_{x}$ the associated system of ordinary differential equations (ODE) is

$$
\frac{d}{d t} \sigma_{x}(t)=F\left(x, \sigma_{x}(t), t\right)
$$

Hamilton's maximum principle is an answer to the following question: For any $t_{0} \in[0, T)$ when will the solution $\sigma(x, t), t \in\left[t_{0}, T\right]$ of the PDE (3) which starts in $\mathcal{K}\left(t_{0}\right)$, remain in $\mathcal{K}(t)$ for all later times, i.e., $\sigma(x, t) \in \mathcal{K}_{x}(t)$ for all $x \in M$ and $t \in\left[t_{0}, T\right]$ ?

In this paper we extend Hamilton's techniques established in [H2] to the case where the convex sets are time-dependent (see Theorem 3 below) and use our extension to also prove a maximum principle for the PDE (3) subject to an avoidance set (see Theorem 4 below). Our first main result is the following:

Theorem 3. Let $\mathcal{K}(t) \subset V, t \in[0, T]$ be closed subsets which satisfy Conditions III and IV above, and such that the space-time track $\mathcal{T}$ is closed. Assume that $u(x, t): V_{x} \times T_{x} M^{*} \rightarrow V_{x}$ is a smooth family of bundle maps of diagonal form and assume that $F(x, \sigma, t)$ is continuous in $x, t$ and is Lipschitz in $\sigma$. Suppose that, for any $x \in M$ and any initial time $t_{0} \in[0, T)$, any solution $\sigma_{x}(t)$ of the $\mathrm{ODE}(4)$ which starts in $\mathcal{K}_{x}\left(t_{0}\right)$ will remain in $\mathcal{K}_{x}(t)$ for all later times, i.e., $\sigma_{x}(t) \in \mathcal{K}_{x}(t)$ for all $t \in\left[t_{0}, T\right]$. Then for any initial time $t_{0} \in[0, T)$ the solution $\sigma(x, t): t \in\left[t_{0}, T\right]$ of the $\mathrm{PDE}(3)$ will remain in $\mathcal{K}(t)$ for all later times if $\sigma(x, t)$ starts in $\mathcal{K}\left(t_{0}\right)$ at time $t_{0}$.

Special cases of this result have been proved by Hamilton and applied to the study of the Ricci flow (see for example Section 2.2 and Section 2.3 in [H5]).

There is also a souped-up version of the maximum principle for systems of reaction-diffusion equations. The idea is that for applications sometimes we are in the situation where the reason that a convex set $\mathcal{K}(t)$ is not preserved 
by the ODE (4) is that the solution wants to escape from a certain part of the convex set (which we will call the avoidance set $\mathcal{A}(t) \subset \mathcal{K}(t)$ ). In this case, if we assume that any solution $\sigma(x, t): t \in\left[t_{0}, T\right]$ to the PDE (3) starts in $\mathcal{K}\left(t_{0}\right) \backslash \mathcal{A}\left(t_{0}\right)$ and assume that the solution $\sigma(x, t)$ does not enter subsets $\mathcal{A}(t)$ for all $t \geq t_{0}$ (i.e., $\sigma(x, t) \notin \mathcal{A}_{x}(t) \doteqdot \mathcal{A}(t) \cap V_{x}$ for all $x \in M$ and all $\left.t \geq t_{0}\right)$, then $\sigma(x, t)$ remains in $\mathcal{K}(t)$ for $t \geq t_{0}$. A typical example where this happens is when the solution to the Ricci flow is assumed not to have any necklike points (see Theorem 3.3 and 3.4 in Section 2.3 of [H5]).

We define the avoidance space-time track

$$
\mathcal{A T} \doteqdot\{(v, t) \in V \times[0, T]: v \in \mathcal{A}(t), t \in[0, T]\},
$$

and define

$$
\mathcal{A} \mathcal{T}_{x} \doteqdot(\mathcal{A T}) \cap\left(V_{x} \times[0, T]\right)
$$

Our second main result is the following:

Theorem 4. Let $\mathcal{K}(t) \subset V, t \in[0, T]$ be closed subsets which satisfy Conditions III and IV above, and such that the space-time and the avoidance space-time tracks $\mathcal{T}$ and $\mathcal{A} \mathcal{T}$ are closed. Assume that $u(x, t): V_{x} \times$ $T_{x} M^{*} \rightarrow V_{x}$ is a smooth family of bundle maps of the diagonal form and that $F(x, \sigma, t)$ is continuous in $x, t$ and is Lipschitz in $\sigma$. Suppose that for any $x \in M, t_{0} \in[0, T)$ and any solution $\sigma_{x}(t)$ of the ODE (4) with initial condition $\sigma_{x}\left(t_{0}\right) \in \mathcal{K}_{x}\left(t_{0}\right) \backslash \mathcal{A}_{x}\left(t_{0}\right)$, either $\sigma_{x}(t) \in \mathcal{K}_{x}(t)$ for all $t \geq t_{0}$, or there is time $t_{1}$ such that $\sigma_{x}(t) \in \mathcal{K}_{x}(t) \backslash \mathcal{A}_{x}(t)$ for all $t_{0} \leq t<t_{1}$ and $\sigma_{x}\left(t_{1}\right) \in \mathcal{A}_{x}\left(t_{1}\right)$. Then for any $t_{0} \in[0, T)$ and any solution $\sigma(x, t): t \in\left[t_{0}, T\right]$ of the PDE (3) satisfying initial condition $\sigma\left(x, t_{0}\right) \in \mathcal{K}_{x}\left(t_{0}\right) \backslash \mathcal{A}_{x}\left(t_{0}\right)$ for all $x \in M$ and satisfying $\sigma(x, t) \notin \mathcal{A}_{x}(t)$ for all $x \in M$ and all $t \geq t_{0}$, the solution $\sigma(x, t)$ will remain in $\mathcal{K}(t)$ for later times.

\section{Hamilton's proof of Theorem 2.}

In order to present the proof of our main result, Theorem 3, more clearly and to exhibit the differences between the proofs of Theorem 3 and Hamilton's Theorem 2, we will first review his proof of Theorem 2 in this section. This will enable us to omit the common parts of the two proofs when we prove Theorem 3.

Theorem 5. Let $\mathcal{K} \subset V$ be a closed subset satisfying Conditions $\mathbf{I}$ and II. Assume that $F(x, \sigma, t)$ is continuous in $x, t$ and is Lipschitz in $\sigma$. Suppose that for any $x \in M$ and any initial time $t_{0} \in[0, T)$, and any solution $\sigma_{x}(t)$ of the ODE (4) which starts in $\mathcal{K}_{x}$ at $t_{0}$, the solution $\sigma_{x}(t)$ will remain in $\mathcal{K}_{x}$ for all later times. Then for any initial time $t_{0} \in[0, T)$ the solution $\sigma(x, t)$ of the $\mathrm{PDE}(3)$ will remain in $\mathcal{K}$ for all later times if $\sigma(x, t)$ starts in $\mathcal{K}$ at time $t_{0}$. 
Remark 6. The above result is slightly more general than Theorem 2 in that it allows for a gradient term in the equation (along the lines of the maximum principle for symmetric 2-tensors in [H1]). This does not affect Hamilton's proof in [H2].

Before proving Theorem 5, we need to recall three lemmas, which are essentially in [H2].

Let $f:[a, b] \rightarrow \mathbb{R}$ be a function. Then we define $\frac{d^{+} f(t)}{d t}$ at $t \in[a, b)$ to be the lim sup of forward difference quotients:

$$
\frac{d^{+} f(t)}{d t}=\limsup _{s \rightarrow 0^{+}} \frac{f(t+s)-f(t)}{s} .
$$

Lemma 7. Suppose function $f:[a, b] \rightarrow \mathbb{R}$ is left lower semi-continuous and right-continuous with $f(a) \leq 0$. Assume either:

(i) $\frac{d^{+} f(t)}{d t} \leq 0$ when $f(t) \geq 0$ on $(a, b)$, or

(ii) for some constant $C<+\infty, \frac{d^{+} f(t)}{d t} \leq C \cdot f(t)$ when $f(t) \geq 0$ on $(a, b)$. Then $f(t) \leq 0$ on $[a, b]$.

Proof. By checking the proof of Lemma 3.1 in [H2], one can prove:

Sublemma: Suppose function $f(t):[a, b] \rightarrow \mathbb{R}$ is left lower semi-continuous and right-continuous with $f(a) \leq 0$. Assume $\frac{d^{+} f(t)}{d t} \leq 0$ when $f(t) \geq 0$ on $[a, b)$. Then $f(t) \leq 0$ on $[a, b]$.

Hypothesis (i) in Lemma 7 is a little weaker than the hypothesis in the sublemma since we do not require the inequality to hold at the left endpoint $a$. By the right continuity at $t=a$, given any $\varepsilon>0$, there exists $\delta>0$ such that $f(t) \leq \varepsilon$ on $[a, a+\delta]$. Now $f(a+\delta)-\varepsilon \leq 0$ and $\frac{d^{+}[f(t)-\varepsilon]}{d t} \leq 0$ when $f(t)-\varepsilon \geq 0$ for $t \in[a+\delta, b)$. Hence we may apply the sublemma to $f(t)-\varepsilon$ to obtain $f(t)-\varepsilon \leq 0$ on $[a+\delta, b]$. We conclude that for any $\varepsilon>0$, we have $f(t) \leq \varepsilon$ for all $t \in[a, b]$. This proves the lemma under Hypothesis (i).

To prove the lemma under Hypothesis (ii), we set $g(t)=e^{-C \cdot t} \cdot f(t)$. Then $\frac{d^{+} g(t)}{d t} \leq 0$ when $g(t) \geq 0$ on $(a, b)$. Applying the lemma under Hypothesis (i) to $g(t)$, we get $g(t) \leq 0$ on $[a, b]$. This implies $f(t) \leq 0$ on $[a, b]$.

The second lemma below gives a useful characterization of when systems of ordinary differential equations preserve closed convex sets in Euclidean space. Let $\mathcal{J} \subset \mathbb{R}^{n}$ be a closed convex subset and $\partial \mathcal{J}$ be the boundary of $\mathcal{J}$ in $\mathbb{R}^{n}$. For any $v \in \partial \mathcal{J}$ we define the tangent cone $C_{v} \mathcal{J}$ of $\mathcal{J}$ at $v$ to be the smallest convex cone in $\mathbb{R}^{n}$ with vertex at $v$ which contains $\mathcal{J}$.

Lemma 8. Let $U \subset \mathbb{R}^{n}$ be an open subset and $\mathcal{J} \subset U$ be a closed convex subset. Consider the ODE

$$
\frac{d \tau}{d t}=F(\tau, t)
$$


where $F: U \times[0, T] \rightarrow \mathbb{R}^{n}$ is continuous in $t$ and is Lipschitz in $\tau$. Then the following two statements are equivalent:

(i) For any initial time $t_{0} \in[0, T)$, any solution of the ODE (6) which starts in $\mathcal{J}$ at $t_{0}$ will remain in $\mathcal{J}$ for all later times;

(ii) $v+F(v, t) \in C_{v} \mathcal{J}$ for all $v \in \partial \mathcal{J}$ and $t \in[0, T)$.

Proof. This is Lemma 4.1 in [H2]. The fact that $F(\tau, t)$ depends on time $t$ does not pose any difficulties for the original proof.

The third lemma gives a general principle on how to take the derivative of a sup-function which plays an important role in proving Theorem 5. Note that $\mathcal{S}$ in the lemma below is independent of time $t$.

Lemma 9. Let $\mathcal{S}$ be a sequentially compact topological space and let $g$ : $\mathcal{S} \times[a, b] \rightarrow \mathbb{R}$ be a function. If $g$ is continuous in $s$ and $t$ and $\frac{\partial g}{\partial t}$ is continuous in $s$ and $t$, then the function $f:[a, b] \rightarrow \mathbb{R}$ defined by

$$
f(t)=\sup _{s \in \mathcal{S}} g(s, t),
$$

is Lipschitz and

$$
\frac{d^{+} f(t)}{d t} \leq \sup \left\{\frac{\partial g}{\partial t}(s, t): s \in \mathcal{S} \text { satisfies } g(s, t)=f(t)\right\} .
$$

Proof. See Lemma 3.5 in [H2].

The rest of this section will be devoted to proving Theorem 5. As remarked in the proof of Lemma 4.1 on p. 160 of [H2] we may assume that $\mathcal{K}$ is compact. For if there were a counterexample $\sigma_{0}(x, t)$ for $t \in\left[t_{0}, T\right]$, then $\sigma_{0}(x, t)$ will be contained in $V(r)$ for some $r$ large enough, where $V(r)$ is the tubular neighborhood of the zero section in $V$ whose intersection with each fiber $V_{x}$ is a ball of radius $r$ around origin measured by metric $h$. Let $\eta$ be a cut-off function on $V$ which equals 1 on $V(r)$ and equals to zero on $V \backslash V(2 r)$. Then we can modify the PDE (3) as

$$
\begin{aligned}
\frac{\partial}{\partial t} \sigma(x, t)= & \Delta(t) \sigma(x, t)+u(x, t)(\nabla(t) \sigma(x, t)) \\
& +\eta(\sigma(x, t)) \cdot F(x, \sigma(x, t), t) .
\end{aligned}
$$

Note that the paths of the counterexample solution $\sigma_{0}(x, t)$ do not change inside $V(r)$, hence $\sigma_{0}(x, t)$ still is a solution of $(7)$. If we intersect $\mathcal{K}$ with $V(2 r)$, we get a counterexample of Theorem 5 for $(7)$ with the closed compact convex set $V(2 r) \cap \mathcal{K} \neq \emptyset$ replacing $\mathcal{K}$, since using Lemma 8 it is easy to check that the ODE

$$
\frac{d}{d t} \sigma_{x}(t)=\eta\left(\sigma_{x}(t)\right) \cdot F\left(x, \sigma_{x}(t), t\right)
$$

and $V(2 r) \cap \mathcal{K}$ satisfy the assumption of Theorem 5 . 
Now we assume that $\mathcal{K}$ is compact. We define the distance between $\sigma \in V_{x}$ and $v \in V_{x}$ using the metric $h$ and denote it by $|\sigma-v|$. We will prove Theorem 5 by contradiction. Suppose we have a solution $\sigma(x, t)$ of the PDE (3) which starts with $\sigma\left(x, t_{0}\right) \in \mathcal{K}_{x}$ for all $x \in M$ and which goes out of $\mathcal{K}$ at some time $t_{2}$. Since $\mathcal{K}$ is closed, we can find a time $t_{1} \geq t_{0}$ such that $\sigma\left(x, t_{1}\right) \in \mathcal{K}_{x}$ for all $x \in M$, and for any $t \in\left(t_{1}, t_{2}\right)$ there is $x$ such that $\sigma(x, t) \notin \mathcal{K}_{x}$. Below we will focus on the time interval $\left[t_{1}, t_{2}\right]$.

Define the function

$$
f(t)=\sup _{x \in M} d\left(\sigma(x, t), \mathcal{K}_{x}\right)=\sup _{x \in M} \inf _{v \in \mathcal{K}_{x}}|\sigma(x, t)-v| \text { for } t \in\left[t_{1}, t_{2}\right] .
$$

We have $f\left(t_{1}\right)=0$ and $f(t)>0$ for $t \in\left(t_{1}, t_{2}\right]$ by assumption. It is easy to check using Condition I that $f(t)$ is a continuous function of $t$. Below we will prove that there is a constant $C<\infty$ such that $\frac{d^{+} f(t)}{d t} \leq C \cdot f(t)$ for $t \in\left(t_{1}, t_{2}\right)$. Once this is proved, then $f(t) \leq 0$ for $t \in\left[t_{1}, t_{2}\right]$ by Lemma 7(ii). Hence $\sigma(x, t) \in \mathcal{K}_{x}$ for all $x \in M$ and all $t \in\left(t_{1}, t_{2}\right]$, we get the required contradiction.

For any $v \in \partial \mathcal{K}_{x}$, let $S_{v} \subset V_{x}$ be the set of outward normal directions $n$ of the supporting hyperplanes of $\mathcal{K}_{x}$ at $v$; we require that $n$ be unit with respect to the metric $h$. Then, since $\mathcal{K}$ is nonempty and for each $t \in\left(t_{1}, t_{2}\right)$, $\sigma(x, t)$ is not in $\mathcal{K}_{x}$ for some $x \in M$, it is well-known that

$$
f(t)=\sup _{x \in M} \sup _{v \in \partial \mathcal{K}_{x}} \sup _{n \in S_{v}} n \cdot(\sigma(x, t)-v),
$$

where is the inner product in $V_{x}$ defined by the metric $h$. Define the set

$$
\mathcal{S}=\left\{(x, v, n): x \in M, v \in \partial \mathcal{K}_{x}, n \in S_{v}\right\}
$$

and the function

$$
g((x, v, n), t)=n \cdot(\sigma(x, t)-v),
$$

then

$$
f(t)=\sup _{(x, v, n) \in \mathcal{S}} g((x, v, n), t) .
$$

Note that $S$ is a compact subset of $V \otimes V$ independent of time $t$, we can apply Lemma 9 and get for any $t \in\left(t_{1}, t_{2}\right)$

$$
\frac{d^{+} f(t)}{d t} \leq \sup \frac{\partial}{\partial t}[n \cdot(\sigma(x, t)-v)]
$$

where the sup is over all $(x, v, n) \in \mathcal{S}$ such that $n \cdot(\sigma(x, t)-v)=f(t)$; in particular we have $|\sigma(x, t)-v|=f(t)$ for these $(x, v, n)$. We compute at 
these $(x, v, n)$

$$
\begin{aligned}
& \frac{\partial}{\partial t}[n \cdot(\sigma(x, t)-v)] \\
& =n \cdot\left(\frac{\partial}{\partial t} \sigma(x, t)\right) \\
& =n \cdot[\Delta(t) \sigma(x, t)]+n \cdot[u(x, t)(\nabla(t) \sigma(x, t))]+n \cdot F(x, \sigma(x, t), t) .
\end{aligned}
$$

By the assumption of Theorem 5 and Lemma 8 we have $v+F(x, v, t) \in C_{v} \mathcal{K}_{x}$. Hence $n \cdot F(x, v, t) \leq 0$ for any $n \in S_{v}$ and any $t \in\left(t_{1}, t_{2}\right)$. We have

$$
\begin{aligned}
& n \cdot F(x, \sigma(x, t), t) \\
& \leq n \cdot F(x, \sigma(x, t), t)-n \cdot F(x, v, t) \\
& \leq n \cdot[F(x, \sigma(x, t), t)-F(x, v, t)] \\
& \leq|F(x, \sigma(x, t), t)-F(x, v, t)| \\
& \leq C \cdot|\sigma(x, t)-v| \\
& =C \cdot f(t),
\end{aligned}
$$

where $C$ is some constant from the assumption that $F(x, \sigma, t)$ is Lipschitz in $\sigma$.

We claim that

$$
\begin{aligned}
n \cdot[u(x, t)(\nabla(t) \sigma(x, t))] & =0, \\
n \cdot[\Delta(t) \sigma(x, t)] & \leq 0,
\end{aligned}
$$

which will be proved in a moment. This shows

$$
\frac{d^{+} f(t)}{d t} \leq C \cdot f(t) \text { on }\left(t_{1}, t_{2}\right)
$$

We are left to prove the claim. We will prove $n \cdot[u(x, t)(\nabla(t) \sigma(x, t))]=0$ and $n \cdot[\Delta(t) \sigma(x, t)] \leq 0$ together. Recall that $(x, v, n)$ satisfies $n \cdot(\sigma(x, t)-$ $v)=f(t)$. If we extend a vector in the bundle $V$ from a point $x$ by parallel translation along geodesics emanating radially out of $x$, we get a smooth section of the bundle on some small neighborhood of $x$ such that all the symmetrized covariant derivatives of the section at $x$ are zero. Let $y$ be an arbitrary point in some small neighborhood $U_{x}$ of $x$. We extend $v \in \partial \mathcal{K}_{x}$ and $n \in V_{x}$ in this manner using the connection $\nabla(t)$ to get $v_{y}$ and $n_{y}$. Since the connection $\nabla(t)$ is compatible with the metric $h$ we continue to have $\left|n_{y}\right|=1$, and since $\mathcal{K}$ is invariant under parallel translation we have $v_{y} \in \partial \mathcal{K}_{y}$ and $n_{y} \in S_{v_{y}}$ for $\mathcal{K}_{y}$ at $v_{y}$. Therefore

$$
n_{y} \cdot\left(\sigma(y, t)-v_{y}\right) \leq f(t)
$$


for all $y \in U_{x}$. It follows that function $n_{y} \cdot\left(\sigma(y, t)-v_{y}\right)$ of $y \in U_{x}$ has a local maximum at $y=x$. So

$$
\begin{gathered}
\frac{\partial}{\partial y^{i}}\left[n_{y} \cdot\left(\sigma(y, t)-v_{y}\right)\right]=0 \text { at } y=x, \\
\Delta(t)\left[n_{y} \cdot\left(\sigma(y, t)-v_{y}\right)\right] \leq 0 \text { at } y=x .
\end{gathered}
$$

Let $\nabla_{t, i}$ be the covariant derivative in direction $\frac{\partial}{\partial y^{i}}$ defined by the connection $\nabla(t)$. Since $v_{y}$ and $n_{y}$ have their symmetrized covariant derivatives equal to zero at $y=x$, so $\nabla_{t, i} n_{y}=\nabla_{t, i} v_{y}=0$ and $\Delta(t) n_{y}=\Delta(t) v_{y}=0$ at $y=x$. Hence

$$
n \cdot\left[\nabla_{t, i} \sigma(x, t)\right]=0, \quad n \cdot[\Delta(t) \sigma(x, t)] \leq 0 .
$$

Then

$$
\begin{aligned}
n \cdot[u(x, t)(\nabla(t) \sigma(x, t))] & =n \cdot\left[\sum_{i} u^{i}(x, t) \cdot \nabla_{t, i} \sigma(x, t)\right] \\
& =\sum_{i} u^{i}(x, t) \cdot\left(n \cdot\left[\nabla_{t, i} \sigma(x, t)\right]\right)=0 .
\end{aligned}
$$

The claim is proved and so is Theorem 5 .

\section{Proof of Theorem 3.}

Throughout this section we will use the same index notation $i$ to denote a sequence or its subsequence or the subsequence of its subsequence. Our arguments below will involve taking subsequences from time to time. The convention will simplify our notations. Before proving Theorem 3, we first formulate a useful characterization of when systems of ordinary differential equations preserve time-dependent closed convex sets in Euclidean space, i.e., a more general version of Lemma 8 .

Let $\mathcal{J}(t) \subset \mathbb{R}^{n}, 0 \leq t \leq T$ be a family of nonempty closed convex subsets. Define the space-time track

$$
\mathcal{L}=\left\{(v, t) \in \mathbb{R}^{n} \times \mathbb{R}: v \in \mathcal{J}(t), 0 \leq t \leq T\right\} .
$$

For each $(v, t) \in \mathcal{L}$ we define a time-like tangent cone in the forward direction of $\mathcal{L}$ at $(v, t)$ and denote it by $C_{(v, t)} \mathcal{L}$. $C_{(v, t)} \mathcal{L}$ consists of all $(W, 1) \in \mathbb{R}^{n} \times$ $\mathbb{R}$ satisfying the following condition: For any sequence $s_{i} \rightarrow 0^{+}$(i.e., $s_{i}$ approaches to zero from positive side), there is a subsequence of $s_{i}$ and vectors $W_{i} \rightarrow W$ such that points $\left(v+s_{i} W_{i}\right) \in \mathcal{J}\left(t+s_{i}\right)$. Note that the definition is stronger than the conventional definition where one sequence of $s_{i}$ is enough. When $\mathcal{J}(t)=\mathcal{J}$ is independent of time $t$, then $C_{(v, t)} \mathcal{L}=$ $\left\{C_{v} \mathcal{J}-v\right\} \times\{1\}$. 
Proposition 10. Let $U \subset R^{n}$ be an open subset and $\mathcal{J}(t) \subset U, 0 \leq t \leq T$ be a family of nonempty closed convex subsets such that the space-time track $\mathcal{L}$ is closed. Consider the ODE

$$
\frac{d \tau}{d t}=F(\tau, t)
$$

where $F: U \times[0, T] \rightarrow \mathbb{R}^{n}$ is continuous in $t$ and is Lipschitz in $\tau$. Then the following two statements are equivalent:

(i) For any initial time $t_{0} \in[0, T)$, any solution of the ODE (9) which starts in $\mathcal{J}\left(t_{0}\right)$ at time $t_{0}$ will remain in $\mathcal{J}(t)$ for all later times;

(ii) $(F(v, t), 1) \in C_{(v, t)} \mathcal{L}$ for all $(v, t) \in \partial \mathcal{L}$, where $\partial \mathcal{L}$ is the boundary of $\mathcal{L} \subset \mathbb{R}^{n+1}$.

Proof. (i) $\Rightarrow$ (ii). For any $\left(v_{0}, t_{0}\right) \in \partial \mathcal{L}$, we consider the solution of (9) with initial condition $\tau\left(t_{0}\right)=v_{0}$. (i) implies that $\tau\left(t_{0}+s\right) \in \mathcal{J}\left(t_{0}+s\right)$ for any $s \in\left[0, T-t_{0}\right]$. Hence

$$
\lim _{s \rightarrow 0^{+}} \frac{\left(\tau\left(t_{0}+s\right), t_{0}+s\right)-\left(\tau\left(t_{0}\right), t_{0}\right)}{s}=\left(F\left(v_{0}, t_{0}\right), 1\right) \in C_{\left(v_{0}, t_{0}\right)} \mathcal{L} .
$$

(ii) $\Rightarrow$ (i). We prove it by contradiction. We will not assume $\mathcal{L}$ to be compact. Suppose we have a solution $\tau(t)$ starting with $\tau\left(t_{0}\right) \in \mathcal{J}\left(t_{0}\right)$ and going out of $\mathcal{L}$ at some time $t_{2}$, i.e., $\tau\left(t_{2}\right) \notin \mathcal{J}\left(t_{2}\right)$. Since $\mathcal{L}$ is closed, we can find a time $t_{1}$ such that $\tau\left(t_{1}\right) \in \mathcal{J}\left(t_{1}\right)$ and $\tau(t) \notin \mathcal{J}(t)$ for all $t \in\left(t_{1}, t_{2}\right)$. Below we will focus on the time interval $\left[t_{1}, t_{2}\right]$.

Let $\partial \mathcal{J}(t)$ be the boundary of $\mathcal{J}(t) \subset \mathbb{R}^{n}$. Define the function

$$
l(t)=d(\tau(t), \mathcal{J}(t)) \text { for } t \in\left[t_{1}, t_{2}\right]
$$

where $d$ is the Euclidean distance on $\mathbb{R}^{n}$. It is clear that $l\left(t_{1}\right)=0$ and $l(t)>0$ for $t \in\left(t_{1}, t_{2}\right]$. Because $\mathcal{L}$ is not assumed to be a domain with smooth boundary, the function $l(t)$ is not necessarily continuous.

Lemma 11. Let $\mathcal{J}(t) \subset U, 0 \leq t \leq T$ be a family of nonempty closed convex subsets. If the space-time track $\mathcal{L}$ is closed and satisfies (ii) in Proposition 10, then $l(t)$ is left lower semi-continuous and is right continuous on $\left[t_{1}, t_{2}\right]$.

Proof of the lemma. To see that $l(t)$ is lower semi-continuous, for any $t \in$ $\left[t_{1}, t_{2}\right]$ and any $s_{i} \rightarrow 0$ with $t+s_{i} \in\left(t_{1}, t_{2}\right]$, we choose $v_{i} \in \partial \mathcal{J}\left(t+s_{i}\right)$ such that

$$
l\left(t+s_{i}\right)=d\left(\tau\left(t+s_{i}\right), v_{i}\right) .
$$

Then either a subsequence $v_{i}$ will converge to some $v_{\infty} \in \mathcal{J}(t)$ since $\mathcal{L}$ is closed, or $v_{i}$ will diverge to $\infty$. In the case of convergence, we have $l\left(t+s_{i}\right) \rightarrow d\left(\tau(t), v_{\infty}\right) \geq l(t)$. The lower semi-continuity is true. In the case of divergence, then $l\left(t+s_{i}\right) \rightarrow+\infty$. Since $\partial \mathcal{J}(t)$ is nonempty, $l(t)$ is finite. Hence the lower semi-continuity of $l(t)$ is also true. 
To prove the right-continuity of $l(t)$, it suffices to prove the upper rightcontinuity. We will use (ii) in Proposition 10 which actually puts some restriction on the space-time track $\mathcal{L}$. It follows from $(\tau(t), t) \notin \mathcal{L}$ for $t>t_{1}$ that $\left(\tau\left(t_{1}\right), t_{1}\right) \in \partial \mathcal{L}$. We denote $\tau\left(t_{1}\right)$ by $v_{t_{1}}$. For any $t \in\left(t_{1}, t_{2}\right)$ it follows from $\tau(t) \notin \mathcal{J}(t)$ that there is $v_{t} \in \partial \mathcal{J}(t)$ such that $l(t)=d\left(\tau(t), v_{t}\right)$. Hence for any $t \in\left[t_{1}, t_{2}\right)$ we can find $v_{t} \in \mathcal{J}(t)$ such that $l(t)=d\left(\tau(t), v_{t}\right)$ and $\left(v_{t}, t\right) \in \partial \mathcal{L}$. By (ii) $\left(F\left(v_{t}, t\right), 1\right) \in C_{\left(v_{t}, t\right)} \mathcal{L}$. If we fix a $t \in\left[t_{1}, t_{2}\right)$, then for any sequence $s_{i} \rightarrow 0^{+}$we can find a subsequence $s_{i}$ such that $\left(v_{t}+s_{i} W_{i}\right) \in \mathcal{J}\left(t+s_{i}\right)$ and $W_{i} \rightarrow F\left(v_{t}, t\right)$. So

$$
l\left(t+s_{i}\right) \leq d\left(\tau\left(t+s_{i}\right), v_{t}+s_{i} W_{i}\right) .
$$

Letting $i \rightarrow \infty$, we get $\lim \sup _{i \rightarrow+\infty} l\left(t+s_{i}\right) \leq d\left(\tau(t), v_{t}\right)=l(t)$. Hence $\lim \sup _{i \rightarrow+\infty} l\left(t+s_{i}\right)=l(t)$ by the lower semi-continuity of $l(\cdot)$. The lemma is proved.

Now we go back to the proof of (ii) $\Rightarrow$ (i) in Proposition 10. Below we will prove that there is some constant $C<\infty$ such that $\frac{d^{+} l(t)}{d t} \leq C \cdot l(t)$ for all $t \in\left(t_{1}, t_{2}\right)$. Once this is proved, then $l(t) \leq 0$ for all $t \in\left[t_{1}, t_{2}\right]$ by Lemma 11 and Lemma 7 . Hence $\tau(t) \in \mathcal{J}(t)$ for $t \in\left[t_{1}, t_{2}\right]$, which is the required contradiction.

Now our proof of the maximum principle with time-dependent convex sets diverges from Hamilton's proof of his maximum principle. This is a necessity in our approach. The key difference is that we will not use the general principle (Lemma 9). We will calculate $\frac{d^{+} l(t)}{d t}$ directly from the definition. Also our proof will not need the cutoff argument which appeared after Lemma 9. For any $t \in\left(t_{1}, t_{2}\right)$ there is a sequence $s_{i} \rightarrow 0^{+}$such that

$$
\frac{d^{+} l(t)}{d t}=\lim _{i \rightarrow \infty} \frac{l\left(t+s_{i}\right)-l(t)}{s_{i}} .
$$

For any $v \in \partial \mathcal{J}(t)$, as in previous section we define $S_{v} \subset \mathbb{R}^{n}$ to be the set of outward normal directions $n$ of the supporting hyperplanes of $\mathcal{J}(t)$ at $v$; we require that $n$ be unit with respect to the Euclidean metric. Define

$$
g(v, n, t)=n \cdot[\tau(t)-v] .
$$

Since $\tau(t) \notin \mathcal{J}(t)$ for $t \in\left(t_{1}, t_{2}\right)$, we have

$$
l(t)=\sup _{v \in \partial \mathcal{J}(t)} \sup _{n \in S_{v}} g(v, n, t)
$$

and so we can find a sequence of points $v_{i} \in \partial \mathcal{J}\left(t+s_{i}\right)$ and $n_{i} \in S_{v_{i}}$ such that $g\left(v_{i}, n_{i}, t+s_{i}\right)=l\left(t+s_{i}\right)=\left|\tau\left(t+s_{i}\right)-v_{i}\right|$. We can also find $v_{\infty} \in \partial \mathcal{J}(t)$ and $n_{\infty} \in S_{v_{\infty}}$ such that $g\left(v_{\infty}, n_{\infty}, t\right)=l(t)=\left|\tau(t)-v_{\infty}\right|$. It is not obvious that such $v_{\infty}$ exists when $t=t_{1}$; this is one of the reason why we use Lemma 7 . The proof below does not need a subsequence of $v_{i}$ to converge to $v_{\infty}$ or a 
subsequence of $n_{i}$ to converge to $n_{\infty}$.

$$
\begin{aligned}
\frac{d^{+} l(t)}{d t} & =\lim _{i \rightarrow \infty} \frac{g\left(v_{i}, n_{i}, t+s_{i}\right)-g\left(v_{\infty}, n_{\infty}, t\right)}{s_{i}} \\
& =\lim _{i \rightarrow \infty} \frac{n_{i} \cdot\left[\tau\left(t+s_{i}\right)-v_{i}\right]-n_{\infty} \cdot\left[\tau(t)-v_{\infty}\right]}{s_{i}} \\
& =\lim _{i \rightarrow \infty} \frac{n_{i} \cdot\left[\tau\left(t+s_{i}\right)-\tau(t)\right]+n_{i} \cdot \tau(t)-n_{i} \cdot v_{i}-n_{\infty} \cdot\left[\tau(t)-v_{\infty}\right]}{s_{i}} .
\end{aligned}
$$

Since $\left(F\left(v_{\infty}, t\right), 1\right) \in C_{\left(v_{\infty}, t\right)} \mathcal{L}$, we can find a subsequence $s_{i}$ and vectors $F_{i} \rightarrow F\left(v_{\infty}, t\right)$ as $i \rightarrow \infty$ such that $\left(v_{\infty}+s_{i} F_{i}\right) \in \mathcal{J}\left(t+s_{i}\right)$. Note that $v_{i} \in \partial \mathcal{J}\left(t+s_{i}\right)$ and $n_{i}$ is the outward normal direction of the supporting hyperplane at $v_{i}$. We have

$$
n_{i} \cdot\left[v_{\infty}+s_{i} F_{i}-v_{i}\right] \leq 0 .
$$

Hence

$$
\begin{aligned}
\frac{d^{+} l(t)}{d t}= & \lim _{i \rightarrow \infty}\left\{n_{i} \cdot\left[\frac{\tau\left(t+s_{i}\right)-\tau(t)}{s_{i}}-F_{i}\right]+\frac{n_{i} \cdot\left[v_{\infty}+s_{i} F_{i}-v_{i}\right]}{s_{i}}\right. \\
& \left.\quad+\frac{\left(n_{i}-n_{\infty}\right) \cdot\left[\tau(t)-v_{\infty}\right]}{s_{i}}\right\} \\
\leq & \lim _{i \rightarrow \infty}\left\{n_{i} \cdot\left[\frac{\tau\left(t+s_{i}\right)-\tau(t)}{s_{i}}-F_{i}\right]+\frac{\left(n_{i}-n_{\infty}\right) \cdot\left[\tau(t)-v_{\infty}\right]}{s_{i}}\right\} \\
\leq & \lim _{i \rightarrow \infty} n_{i} \cdot\left[\frac{\tau\left(t+s_{i}\right)-\tau(t)}{s_{i}}-F_{i}\right] \\
\leq & \lim _{i \rightarrow \infty}\left|\frac{\tau\left(t+s_{i}\right)-\tau(t)}{s_{i}}-F_{i}\right| \\
= & \left|F(\tau(t), t)-F\left(v_{\infty}, t\right)\right| \\
\leq & C \cdot\left|\tau(t)-v_{\infty}\right| \\
= & C \cdot l(t) .
\end{aligned}
$$

We have used $\left(n_{i}-n_{\infty}\right) \cdot\left[\tau(t)-v_{\infty}\right] \leq 0$ to get the second inequality above. This is because $n_{i} \cdot\left[\tau(t)-v_{\infty}\right] \leq\left|\tau(t)-v_{\infty}\right|$ and $\left|\tau(t)-v_{\infty}\right|=n_{\infty} \cdot\left[\tau(t)-v_{\infty}\right]$. We have used $\left|n_{i}\right|=1$ to get the third inequality above. Proposition 10 is now proved.

The rest of this section is devoted to the proof of Theorem 3. We will prove it by contradiction. Suppose we have a solution $\sigma(x, t)$ of the PDE (3) on $\left[t_{0}, T\right]$ which starts with $\sigma\left(x, t_{0}\right) \in \mathcal{K}_{x}\left(t_{0}\right)$ for all $x \in M$ and which goes out of space-time track $\mathcal{T}$ at some time $t_{2}$. Since $\mathcal{T}$ is closed, there is a time $t_{1} \geq t_{0}$ such that $\sigma\left(x, t_{1}\right) \in \mathcal{K}_{x}\left(t_{1}\right)$ for all $x \in M$ and for any $t_{1}<t<t_{2}$ there is $x$ such that $\sigma(x, t) \notin \mathcal{K}_{x}(t)$. Below we will focus on the time interval $\left[t_{1}, t_{2}\right]$. 
Define the function

$$
f(t)=\sup _{x \in M} d\left(\sigma(x, t), \mathcal{K}_{x}(t)\right) \text { for } t \in\left[t_{1}, t_{2}\right]
$$

where $d$ is distance on $V_{x}$ defined by the metric $h$. It is clear from our choice that $f\left(t_{1}\right)=0, f(t)>0$ for $t>t_{1}$. Note that $f(t)$ is not necessarily continuous.

Next we prove a lemma which will enable us later to apply Lemma 7 to $f(t)$ defined by (10). Let $\hat{\sigma}(x, t)$ be any continuous section of bundle $V$ which satisfies that $\hat{\sigma}\left(x, t_{1}\right) \in \mathcal{K}_{x}\left(t_{1}\right)$ for all $x \in M$ and where for each $t \in\left(t_{1}, t_{2}\right]$ there is $x$ such that $\hat{\sigma}(x, t)$ is not in $\mathcal{K}_{x}(t)$. We define the function $\widehat{g}: M \times\left[t_{1}, t_{2}\right] \rightarrow \mathbb{R}$ by

$$
\widehat{g}(x, t)=d\left(\hat{\sigma}(x, t), \mathcal{K}_{x}(t)\right),
$$

and define the function

$$
\hat{f}(t)=\sup _{x \in M} \widehat{g}(x, t) \text { for } t \in\left[t_{1}, t_{2}\right] .
$$

By assumption $\hat{f}\left(t_{1}\right)=0$, and $\hat{f}(t)>0$ for $t \in\left(t_{1}, t_{2}\right]$. For any $t \in\left[t_{1}, t_{2}\right)$ and any sequence $s_{i} \rightarrow 0^{+}$, there is a subsequence $s_{i}$ and a sequence $x_{i} \in M$ such that $\widehat{g}\left(x_{i}, t+s_{i}\right)=\sup _{x \in M} \widehat{g}\left(x, t+s_{i}\right)$ and $x_{i} \rightarrow x_{\infty}$.

Lemma 12. For the space-time track $\mathcal{T}$ satisfying the assumption of Theorem $3, \hat{f}(t)$ is left lower semi-continuous and is right-continuous on $\left[t_{1}, t_{2}\right]$, and for $t \in\left[t_{1}, t_{2}\right)$ the above chosen $x_{\infty}$ satisfies

$$
\widehat{g}\left(x_{\infty}, t\right)=\hat{f}(t) .
$$

Proof of the lemma. First we show that $\widehat{f}(t)$ is lower semi-continuous. $\widehat{f}(t)$ is obviously lower semi-continuous at $t=t_{1}$. At any $t=t_{a} \in\left(t_{1}, t_{2}\right]$, we have $\widehat{f}\left(t_{a}\right)>0$. We fix $x_{a}$ such that $\widehat{f}\left(t_{a}\right)=\widehat{g}\left(x_{a}, t_{a}\right)$. Then since $\mathcal{T}$ is closed, there is an $\varepsilon>0$ such that $\hat{\sigma}\left(x_{a}, t\right) \notin \mathcal{K}_{x_{a}}(t)$ for $t \in\left(t_{a}-\varepsilon, t_{a}+\varepsilon\right)$. We can apply Lemma 11 to $\widehat{g}\left(x_{a}, t\right)$ in the fiber $V_{x_{a}}$ to conclude that $\widehat{g}\left(x_{a}, \cdot\right)$ is lower semi-continuous at $t=t_{a}$. Hence for any $s_{i} \rightarrow 0$

$$
\lim \inf _{i \rightarrow+\infty} \widehat{f}\left(t_{a}+s_{i}\right) \geq \lim \inf _{i \rightarrow+\infty} \widehat{g}\left(x_{a}, t_{a}+s_{i}\right) \geq \widehat{g}\left(x_{a}, t_{a}\right)=\widehat{f}\left(t_{a}\right) .
$$

Hence $\widehat{f}(t)$ is lower semi-continuous at time $t=t_{a}$, and hence on $\left[t_{1}, t_{2}\right]$.

To prove the right-continuity of $\widehat{f}(t)$, it suffices to prove the upper rightcontinuity. For any $t_{a} \in\left[t_{1}, t_{2}\right)$ and any sequence $s_{i} \rightarrow 0^{+}$we will show that there is a subsequence $s_{i}$ such that $\lim _{i \rightarrow \infty} \widehat{f}\left(t_{a}+s_{i}\right) \leq \widehat{f}\left(t_{a}\right)$. By passing to a subsequence if necessarily we may assume that $\lim _{i \rightarrow \infty} \widehat{f}\left(t_{a}+s_{i}\right)$ exists. Choose $x_{i} \in M$ satisfying $\widehat{f}\left(t_{a}+s_{i}\right)=\widehat{g}\left(x_{i}, t_{a}+s_{i}\right)$; without loss of generality, we may assume that $x_{i} \rightarrow x_{\infty}$ by taking a subsequence if necessary. Let $v_{\infty} \in \mathcal{K}_{x_{\infty}}\left(t_{a}\right)$ such that $\widehat{g}\left(x_{\infty}, t_{a}\right)=d\left(\widehat{\sigma}\left(x_{\infty}, t_{a}\right), v_{\infty}\right)$. It follows from $\widehat{f}\left(t_{a}+s_{i}\right)>0$, the invariance of $\mathcal{T}_{x}$ under parallel translation and 
the closedness of $\mathcal{T}$, that $\left(v_{\infty}, t_{a}\right) \in \partial \mathcal{T}_{x_{\infty}}$. By the assumption of Theorem 3 and Proposition 10, $C_{\left(v_{\infty}, t_{a}\right)} \mathcal{T}_{x_{\infty}}$ is nonempty. Then there is a subsequence $\left(v_{\infty}+s_{i} W_{i}\right) \in \mathcal{K}_{x_{\infty}}\left(t_{a}+s_{i}\right)$ with $W_{i} \rightarrow W$ for some $W \in V_{x_{\infty}}$. Hence

$$
d\left(\widehat{\sigma}\left(x_{\infty}, t_{a}+s_{i}\right), v_{\infty}+s_{i} W_{i}\right) \geq d\left(\widehat{\sigma}\left(x_{\infty}, t_{a}+s_{i}\right), \mathcal{K}_{x_{\infty}}\left(t_{a}+s_{i}\right)\right) .
$$

Since $\widehat{\sigma}\left(x, t_{a}\right)$ is continuous in $x$ and $\mathcal{K}_{x}\left(t_{a}+s_{i}\right)$ is invariant under parallel translation $\nabla\left(t_{a}+s_{i}\right)$ for any $x \in M, d\left(\widehat{\sigma}\left(x_{\infty}, t_{a}+s_{i}\right), \mathcal{K}_{x_{\infty}}\left(t_{a}+s_{i}\right)\right)$ can be chosen arbitrarily close to $d\left(\widehat{\sigma}\left(x_{i}, t_{a}+s_{i}\right), \mathcal{K}_{x_{i}}\left(t_{a}+s_{i}\right)\right)=\widehat{g}\left(x_{i}, t_{a}+s_{i}\right)$ when $i$ is large, so the right side of (11) approaches $\lim _{i \rightarrow+\infty} \widehat{f}\left(t_{a}+s_{i}\right)$. The left side of (11) approaches

$$
d\left(\widehat{\sigma}\left(x_{\infty}, t_{a}\right), v_{\infty}\right)=d\left(\widehat{\sigma}\left(x_{\infty}, t_{a}\right), \mathcal{K}_{x_{\infty}}\left(t_{a}\right)\right) \leq \widehat{f}\left(t_{a}\right) .
$$

Now we have proved $\lim _{i \rightarrow+\infty} \widehat{f}\left(t_{a}+s_{i}\right) \leq \widehat{f}\left(t_{a}\right)$ and hence the right continuity of $\widehat{f}(t)$.

By taking the limit of (11) we have

$$
\widehat{g}\left(x_{\infty}, t_{a}\right)=d\left(\widehat{\sigma}\left(x_{\infty}, t_{a}\right), \mathcal{K}_{x_{\infty}}\left(t_{a}\right)\right) \geq \lim _{i \rightarrow+\infty} \widehat{f}\left(t_{a}+s_{i}\right) .
$$

Since $\widehat{f}(t)$ is right-continuous and $\widehat{g}\left(x_{\infty}, t_{a}\right) \leq \widehat{f}\left(t_{a}\right)$, we conclude that $\widehat{g}\left(x_{\infty}, t_{a}\right)=\widehat{f}\left(t_{a}\right)$ for any $t_{a} \in\left[t_{1}, t_{2}\right)$. The lemma is proved.

Now we go back to the proof of Theorem 3. Let $f(t)$ be the function defined in (10), we will prove that there is a constant $C<+\infty$ such that $\frac{d^{+} f(t)}{d t} \leq C \cdot f(t)$ for $t \in\left(t_{1}, t_{2}\right)$. Once this is proved, from Lemma 12 and Lemma 7 we conclude that $f(t)=0$ for $t \in\left[t_{1}, t_{2}\right]$, and hence $\sigma(x, t) \in \mathcal{K}_{x}(t)$ for all $x \in M$ and $t \in\left[t_{1}, t_{2}\right]$. We get the required contradiction.

For any $t_{a} \in\left(t_{1}, t_{2}\right)$ there exists a sequence $s_{i} \rightarrow 0^{+}$such that

$$
\frac{d^{+} f\left(t_{a}\right)}{d t}=\lim _{i \rightarrow \infty} \frac{f\left(t_{a}+s_{i}\right)-f\left(t_{a}\right)}{s_{i}} .
$$

We define the function

$$
g(x, v, n, t)=n \cdot[\sigma(x, t)-v] \text {, for } x \in M, n \in V_{x}, v \in V_{x} \text {, and } t \in\left[t_{1}, t_{2}\right] .
$$

For any $v \in \partial \mathcal{K}_{x}(t)$, we define $S_{v} \subset V_{x}$ to be the set of the outward unit normal directions $n$ of the supporting hyperplanes of $\mathcal{K}_{x}(t)$ in $V_{x}$ at $v$. Then, for any $t>t_{1}$ since $\mathcal{K}_{x}(t)$ is not empty and $\sigma(x, t)$ is not in the interior of $\mathcal{K}_{x}(t)$ for some $x \in M$, it is well-known that

$$
f(t)=\sup _{x \in M} \sup _{v \in \partial \mathcal{K}_{x}(t)} \sup _{n \in S_{v}} g(x, v, n, t) .
$$

Note that the set over which we take the supremum in the definition of $f(t)$ depends on time. This is why we compute $\frac{d^{+} f\left(t_{a}\right)}{d t}$ directly rather than using Lemma 9.

We can find a sequence of points $x_{i} \in M, v_{i} \in \partial \mathcal{K}_{x_{i}}\left(t_{a}+s_{i}\right)$, and $n_{i} \in S_{v_{i}}$, such that $g\left(x_{i}, v_{i}, n_{i}, t_{a}+s_{i}\right)=f\left(t_{a}+s_{i}\right)$, by Lemma 12 we may assume $x_{i} \rightarrow$ 
$x_{\infty} \in M$ and $f\left(t_{a}\right)=d\left(\sigma\left(x_{\infty}, t_{a}\right), \mathcal{K}_{x_{\infty}}\left(t_{a}\right)\right)$. Since $t_{a}>t_{1}$ and $f\left(t_{a}\right)>0$, we have $f\left(t_{a}\right)=\sup _{v \in \partial \mathcal{K}_{x_{\infty}}\left(t_{a}\right)} \sup _{n \in S_{v}} g\left(x_{\infty}, v, n, t_{a}\right)$. Let $v_{\infty} \in \partial \mathcal{K}_{x_{\infty}}\left(t_{a}\right)$ and $n_{\infty} \in S_{v_{\infty}}$, such that $g\left(x_{\infty}, v_{\infty}, n_{\infty}, t_{a}\right)=f\left(t_{a}\right)$.

We claim that there is a subsequence $i$ such that $v_{i} \rightarrow v_{\infty}$ and $n_{i} \rightarrow n_{\infty}$ in the bundle $V$. Since $d\left(\sigma\left(x_{i}, t_{a}+s_{i}\right), v_{i}\right)=f\left(t_{a}+s_{i}\right)$ and $f\left(t_{a}+s_{i}\right)$ is uniformly bounded from above by the right-continuity of $f(t)$, we can rule out the divergence of $v_{i}$ to $\infty$. We may assume that there is a subsequence $i$ such that $v_{i} \rightarrow \widehat{v}_{\infty}$ and $n_{i} \rightarrow \widehat{n}_{\infty}$. By the closedness of $\mathcal{T}$ we have $\hat{v}_{\infty} \in \mathcal{K}_{x_{\infty}}\left(t_{a}\right)$, also we have $\left|\widehat{n}_{\infty}\right|=1$. By taking the limit of

$$
n_{i} \cdot\left[\sigma\left(x_{i}, t_{a}+s_{i}\right)-v_{i}\right]=f\left(t_{a}+s_{i}\right), \quad\left|\sigma\left(x_{i}, t_{a}+s_{i}\right)-v_{i}\right|=f\left(t_{a}+s_{i}\right),
$$

we get

$$
\begin{aligned}
\widehat{n}_{\infty} \cdot\left[\sigma\left(x_{\infty}, t_{a}\right)-\widehat{v}_{\infty}\right] & =f\left(t_{a}\right), \\
\left|\sigma\left(x_{\infty}, t_{a}\right)-\widehat{v}_{\infty}\right| & =f\left(t_{a}\right) .
\end{aligned}
$$

By the convexity of $\mathcal{K}_{x_{\infty}}\left(t_{a}\right)$, (13) implies $\widehat{v}_{\infty}=v_{\infty}$ and (12) implies $\widehat{n}_{\infty}=$ $n_{\infty}$. The claim is proved.

Then

$$
\begin{aligned}
\frac{d^{+} f\left(t_{a}\right)}{d t}= & \lim _{i \rightarrow \infty} \frac{g\left(x_{i}, v_{i}, n_{i}, t_{a}+s_{i}\right)-g\left(x_{\infty}, v_{\infty}, n_{\infty}, t_{a}\right)}{s_{i}} \\
= & \lim _{i \rightarrow \infty} \frac{n_{i} \cdot\left[\sigma\left(x_{i}, t_{a}+s_{i}\right)-v_{i}\right]-n_{\infty} \cdot\left[\sigma\left(x_{\infty}, t_{a}\right)-v_{\infty}\right]}{s_{i}} \\
= & \lim _{i \rightarrow \infty}\left\{\frac{n_{i} \cdot\left[\sigma\left(x_{i}, t_{a}+s_{i}\right)-\sigma\left(x_{i}, t_{a}\right)\right]+n_{i} \cdot \sigma\left(x_{i}, t_{a}\right)}{s_{i}}\right. \\
& \left.+\frac{-n_{i} \cdot v_{i}-n_{\infty} \cdot\left[\sigma\left(x_{\infty}, t_{a}\right)-v_{\infty}\right]}{s_{i}}\right\} .
\end{aligned}
$$

To estimate quantities at $\left(x_{i}, t_{a}+s_{i}\right)$ and at $\left(x_{\infty}, t_{a}\right)$ in $\frac{d^{+} f\left(t_{a}\right)}{d t}$, we interpose quantities at $\left(x_{i}, t_{a}\right)$ (see (14) below). By Lemma 12, $d\left(\sigma\left(x_{\infty}, t_{a}\right)\right.$, $\left.\mathcal{K}_{x_{\infty}}\left(t_{a}\right)\right)=f\left(t_{a}\right)>0$, and hence $\sigma\left(x_{\infty}, t_{a}\right) \notin \mathcal{K}_{x_{\infty}}\left(t_{a}\right)$. It follows from $\mathcal{K}_{x}\left(t_{a}\right)$ being invariant under parallel translation that for large enough $i$, $\sigma\left(x_{i}, t_{a}\right) \notin \mathcal{K}_{x_{i}}\left(t_{a}\right)$. We can choose $v_{i}^{*} \in \partial \mathcal{K}_{x_{i}}\left(t_{a}\right)$ and $n_{i}^{*} \in S_{v_{i}^{*}}$ such that $d\left(\sigma\left(x_{i}, t_{a}\right), \mathcal{K}_{x_{i}}\left(t_{a}\right)\right)=n_{i}^{*} \cdot\left[\sigma\left(x_{i}, t_{a}\right)-v_{i}^{*}\right]$. Such $v_{i}^{*}$ and $n_{i}^{*}$ may not exist at time $t_{a}=t_{1}$ since $\sigma\left(x_{\infty}, t_{1}\right) \in \mathcal{K}_{x_{\infty}}\left(t_{1}\right)$; this is another reason why we need Lemma 7.

We claim that there is a sequence of vectors $F_{i} \in V_{x_{i}}$ such that for any $\varepsilon>$ 0 there is an $i_{0}$ such that for any $i \geq i_{0}$ we have $v_{i}^{*}+s_{i} F_{i} \in \mathcal{K}_{x_{i}}\left(t_{a}+s_{i}\right)$ and $\left|F_{i}-F\left(x_{i}, v_{i}^{*}, t_{a}\right)\right| \leq \varepsilon$. The claim can be proved by studying a family indexed by $i$ of ODE (4) in $V_{x_{i}}$ with initial time $t_{a}$ and initial value $\sigma_{x_{i}}\left(t_{a}\right)=v_{i}^{*}$. We write the solution $\sigma_{x_{i}}\left(t_{a}+s_{i}\right)=v_{i}^{*}+s_{i} F_{i}$. It follows from the assumption of Theorem 3 that $\sigma_{x_{i}}\left(t_{a}+s_{i}\right) \in \mathcal{K}_{x_{i}}\left(t_{a}+s_{i}\right)$. Since $F(x, \sigma, t)$ is Lipschitz in $\sigma$, the inequality $\left|F_{i}-F\left(x_{i}, v_{i}^{*}, t_{a}\right)\right| \leq \varepsilon$ follows from the fact that solutions of 
ordinary differential equations depend continuously on their parameters, in this case the parameters are $x_{i} \in M$ and $v_{i}^{*} \in \partial \mathcal{K}_{x_{i}}\left(t_{a}\right)$ varying in compact domain.

Since $d\left(\sigma\left(x_{i}, t_{a}\right), \mathcal{K}_{x_{i}}\left(t_{a}\right)\right)=d\left(\sigma\left(x_{i}, t_{a}\right), v_{i}^{*}\right) \leq f\left(t_{a}\right)<\infty$, we can rule out the divergence of $v_{i}^{*}$ to $\infty$. We may assume that a subsequence $v_{i}^{*}$ converges to $v_{\infty}^{*} \in V_{x_{\infty}}$, and get $d\left(\sigma\left(x_{\infty}, t_{a}\right), \mathcal{K}_{x_{\infty}}\left(t_{a}\right)\right)=d\left(\sigma\left(x_{\infty}, t_{a}\right), v_{\infty}^{*}\right)$. By the closedness of the space-time track $\mathcal{T}$ we have $v_{\infty}^{*} \in \mathcal{K}_{x_{\infty}}\left(t_{a}\right)$. Since

$$
d\left(\sigma\left(x_{\infty}, t_{a}\right), v_{\infty}^{*}\right)=d\left(\sigma\left(x_{\infty}, t_{a}\right), \mathcal{K}_{x_{\infty}}\left(t_{a}\right)\right)=d\left(\sigma\left(x_{\infty}, t_{a}\right), v_{\infty}\right)
$$

and $\mathcal{K}_{x_{\infty}}\left(t_{a}\right)$ is convex, we conclude that $v_{\infty}^{*}=v_{\infty}$. Our choice of $F_{i}$ ensures that $\lim _{i \rightarrow \infty} F_{i}=F\left(x_{\infty}, v_{\infty}^{*}, t_{a}\right)=F\left(x_{\infty}, v_{\infty}, t_{a}\right)$. Recall that $v_{i} \in \partial \mathcal{K}_{x_{i}}\left(t_{a}+\right.$ $s_{i}$ ) and $n_{i}$ is the outward normal direction of the supporting hyperplane at $v_{i}$. We have in each fiber $V_{x_{i}}$ and at time $t_{a}+s_{i}$

$$
n_{i} \cdot\left[v_{i}^{*}+s_{i} F_{i}-v_{i}\right] \leq 0 .
$$

Hence

$$
\begin{aligned}
\frac{d^{+} f\left(t_{a}\right)}{d t}= & \lim _{i \rightarrow \infty}\left\{n_{i} \cdot\left[\frac{\sigma\left(x_{i}, t_{a}+s_{i}\right)-\sigma\left(x_{i}, t_{a}\right)}{s_{i}}-F_{i}\right]\right. \\
& +\frac{n_{i} \cdot\left[v_{i}^{*}+s_{i} F_{i}-v_{i}\right]}{s_{i}} \\
& \left.+\frac{n_{i} \cdot\left[\sigma\left(x_{i}, t_{a}\right)-v_{i}^{*}\right]-n_{\infty} \cdot\left[\sigma\left(x_{\infty}, t_{a}\right)-v_{\infty}\right]}{s_{i}}\right\} \\
\leq & \lim _{i \rightarrow \infty}\left\{n_{i} \cdot\left[\frac{\sigma\left(x_{i}, t_{a}+s_{i}\right)-\sigma\left(x_{i}, t_{a}\right)}{s_{i}}-F_{i}\right]\right. \\
& \left.+\frac{n_{i} \cdot\left[\sigma\left(x_{i}, t_{a}\right)-v_{i}^{*}\right]-n_{\infty} \cdot\left[\sigma\left(x_{\infty}, t_{a}\right)-v_{\infty}\right]}{s_{i}}\right\} \\
\leq & \lim _{i \rightarrow \infty}\left\{n_{i} \cdot\left[\frac{\sigma\left(x_{i}, t_{a}+s_{i}\right)-\sigma\left(x_{i}, t_{a}\right)}{s_{i}}-F_{i}\right]\right\}
\end{aligned}
$$

where to get the last inequality above we have used

$$
n_{i} \cdot\left[\sigma\left(x_{i}, t_{a}\right)-v_{i}^{*}\right] \leq n_{\infty} \cdot\left[\sigma\left(x_{\infty}, t_{a}\right)-v_{\infty}\right] .
$$

This is because

$$
n_{i} \cdot\left[\sigma\left(x_{i}, t_{a}\right)-v_{i}^{*}\right] \leq\left|\sigma\left(x_{i}, t_{a}\right)-v_{i}^{*}\right|=d\left(\sigma\left(x_{i}, t_{a}\right), \mathcal{K}_{x_{i}}\left(t_{a}\right)\right),
$$

and at time $t_{a}$

$$
\begin{aligned}
d\left(\sigma\left(x_{i}, t_{a}\right), \mathcal{K}_{x_{i}}\left(t_{a}\right)\right) \leq f\left(t_{a}\right) & =d\left(\sigma\left(x_{\infty}, t_{a}\right), \mathcal{K}_{x_{\infty}}\left(t_{a}\right)\right) \\
& =n_{\infty} \cdot\left[\sigma\left(x_{\infty}, t_{a}\right)-v_{\infty}\right]
\end{aligned}
$$


by our choice of $x_{\infty}, v_{\infty}$, and $n_{\infty}$.

$$
\begin{aligned}
\frac{d^{+} f\left(t_{a}\right)}{d t} \leq & {\left[\lim _{i \rightarrow \infty} n_{i}\right] \cdot\left[\lim _{i \rightarrow \infty} \frac{\sigma\left(x_{i}, t_{a}+s_{i}\right)-\sigma\left(x_{i}, t_{a}\right)}{s_{i}}-\lim _{i \rightarrow \infty} F_{i}\right] } \\
= & n_{\infty} \cdot\left[\frac{\partial}{\partial t} \sigma\left(x_{\infty}, t_{a}\right)-F\left(x_{\infty}, v_{\infty}, t_{a}\right)\right] \\
= & n_{\infty} \cdot\left[\Delta\left(t_{a}\right) \sigma\left(x_{\infty}, t_{a}\right)+u\left(x_{\infty}, t_{a}\right)\left(\nabla\left(t_{a}\right) \sigma\left(x_{\infty}, t_{a}\right)\right)\right. \\
& \left.+F\left(x_{\infty}, \sigma\left(x_{\infty}, t_{a}\right), t_{a}\right)-F\left(x_{\infty}, v_{\infty}, t_{a}\right)\right] \\
= & n_{\infty} \cdot\left[\Delta\left(t_{a}\right) \sigma\left(x_{\infty}, t_{a}\right)\right]+n_{\infty} \cdot\left[u\left(x_{\infty}, t_{a}\right)\left(\nabla\left(t_{a}\right) \sigma\left(x_{\infty}, t_{a}\right)\right)\right] \\
& +n_{\infty} \cdot\left[F\left(x_{\infty}, \sigma\left(x_{\infty}, t_{a}\right), t_{a}\right)-F\left(x_{\infty}, v_{\infty}, t_{a}\right)\right] .
\end{aligned}
$$

By the same argument as in Section 3 we conclude that

$$
\begin{aligned}
n_{\infty} \cdot\left[\Delta\left(t_{a}\right) \sigma\left(x_{\infty}, t_{a}\right)\right] & \leq 0, \\
n_{\infty} \cdot\left[u\left(x_{\infty}, t_{a}\right)\left(\nabla\left(t_{a}\right) \sigma\left(x_{\infty}, t_{a}\right)\right)\right] & =0 .
\end{aligned}
$$

So

$$
\begin{aligned}
\frac{d^{+} f\left(t_{a}\right)}{d t} & \leq n_{\infty} \cdot\left[F\left(x_{\infty}, \sigma\left(x_{\infty}, t_{a}\right), t_{a}\right)-F\left(x_{\infty}, v_{\infty}, t_{a}\right)\right] \\
& \leq\left|F\left(x_{\infty}, \sigma\left(x_{\infty}, t_{a}\right), t_{a}\right)-F\left(x_{\infty}, v_{\infty}, t_{a}\right)\right| \\
& \leq C \cdot\left|\sigma\left(x_{\infty}, t_{a}\right)-v_{\infty}\right| \\
& =C \cdot f\left(t_{a}\right) .
\end{aligned}
$$

Theorem 3 is proved.

\section{Proof of Theorem 4.}

First we prove a version of Proposition 10 subject to an avoidance set.

Proposition 13. Let $U \subset \mathbb{R}^{n}$ be an open subset, $\mathcal{J}(t) \subset U, t \in[0, T]$ be a family of nonempty closed convex subsets and $\mathcal{B}(t) \subset \mathcal{J}(t)$ be avoidance sets such that the space-time track $\mathcal{L}$ and the avoidance space-time track $\mathcal{B L}=\left\{(v, t) \in \mathbb{R}^{n} \times \mathbb{R}: v \in \mathcal{B}(t), t \in[0, T]\right\}$ are closed. Consider the ODE

$$
\frac{d \tau}{d t}=F(\tau, t)
$$

where $F: U \times[0, T] \rightarrow \mathbb{R}^{n}$ is continuous in $t$ and is Lipschitz in $\tau$. Then the following two statements are equivalent:

(i) For any $t_{0} \in[0, T)$ and any solution $\tau(t), t \in\left[t_{0}, T\right]$ of the ODE (15) with initial condition $\tau\left(t_{0}\right) \in \mathcal{J}\left(t_{0}\right) \backslash \mathcal{B}\left(t_{0}\right)$, either $\tau(t) \in \mathcal{J}(t)$ for all $t \geq t_{0}$, or there is a time $t_{1}>t_{0}$ such that $\tau(t) \in \mathcal{J}(t) \backslash \mathcal{B}(t)$ for all $t \in\left[t_{0}, t_{1}\right)$ and $\tau\left(t_{1}\right) \in \mathcal{B}\left(t_{1}\right)$.

(ii) $(F(v, t), 1) \in C_{(v, t)} \mathcal{L}$ for all $(v, t) \in(\partial \mathcal{L}) \backslash(\mathcal{B L})$. 
Proof. This proposition can be proved as Proposition 10 except for the following issue which arises in proving (ii) $\Longrightarrow(\mathrm{i})$ : In the proof of Proposition 10 we have used the property $(F(v, t), 1) \in C_{(v, t)} \mathcal{L}$ for all $(v, t) \in \partial \mathcal{L}$, however here this property holds only for $(v, t) \in(\partial \mathcal{L}) \backslash(\mathcal{B L})$. We need to ensure that $(v, t)$ can be chosen in $(\partial \mathcal{L}) \backslash(\mathcal{B L})$ when we use this property in the proof of Proposition 10.

We adopt the notations used in the proof of Proposition 10 and resolve the issue. Since $\mathcal{B L}$ is closed and the solution $\tau(t), t \in\left[t_{1}, t_{2}\right]$ in the proof of Lemma 11 does not enter in $\mathcal{B L}$, there is a constant $\varepsilon>0$ such that

$$
\inf _{t \in\left[t_{1}, t_{2}\right]} d(\tau(t), \mathcal{B}(t)) \geq 3 \varepsilon .
$$

Since $\left(v_{t_{1}}, t_{1}\right)=\left(\tau\left(t_{1}\right), t_{1}\right) \in(\partial \mathcal{L}) \backslash(\mathcal{B L}), l(t)$ is right-continuous at $t_{1}$ by the proof of Lemma 11. Hence there is $t_{3} \in\left(t_{1}, t_{2}\right)$ such that $f(t) \leq \varepsilon$ for all $t \in\left(t_{1}, t_{3}\right)$. For any $t \in\left(t_{1}, t_{3}\right)$

$$
d\left(v_{t}, \mathcal{B}(t)\right) \geq d(\tau(t), \mathcal{B}(t))-d\left(v_{t}, \tau(t)\right) \geq 2 \varepsilon,
$$

hence $\left(v_{t}, t\right) \in(\partial \mathcal{L}) \backslash(\mathcal{B L})$ for all $t \in\left(t_{1}, t_{3}\right)$ and again $l(t)$ can be shown to be left lower semi-continuous and right-continuous on $\left[t_{1}, t_{3}\right]$.

For any $t \in\left(t_{1}, t_{3}\right)$, choose the points $\left(v_{\infty}, t\right)$ in $\partial \mathcal{L}$ as in the proof of Proposition 10. These points are at least $2 \varepsilon$ away from $\mathcal{B L}$, so by Statement (ii) we still have the property $\left(F\left(v_{\infty}, t\right), 1\right) \in C_{\left(v_{\infty}, t\right)} \mathcal{L}$, which was use in the proof of Proposition 10. We may now repeat the rest of the proof of Proposition 10 to conclude that there is a constant $C<+\infty$ such that $\frac{d^{+} f(t)}{d t} \leq C \cdot f(t)$ for all $t \in\left(t_{1}, t_{3}\right)$. By Lemma 7 we get $l(t)=0$ on $\left[t_{1}, t_{3}\right]$, which is the required contradiction.

The intuition behind the proof of Theorem 4 is as follows: Outside the avoidance set (where the solution is assumed not to enter) the reaction term of the PDE (i.e., corresponding to the associated ODE) wants to push the solution back into the convex set. The diffusion part wants to keep the solution in the convex set, possibly trying (but not succeeding) to push it into the avoidance part.

Proof of Theorem 4. We will prove it by contradiction. As in the proof of Theorem 3, suppose we have a solution $\sigma(x, t)$ of $\mathrm{PDE}(3)$ on $\left[t_{0}, T\right]$ which starts with $\sigma\left(x, t_{0}\right) \in \mathcal{K}_{x}\left(t_{0}\right) \backslash \mathcal{A}_{x}\left(t_{0}\right)$ for all $x \in M$ and which goes out of the space-time track $\mathcal{T}$ at some time $t_{2}$. Since $\mathcal{T}$ is closed, there is a time $t_{1} \geq t_{0}$ such that $\sigma\left(x, t_{1}\right) \in \mathcal{K}_{x}\left(t_{1}\right)$ for all $x$ and for any $t \in\left(t_{1}, t_{2}\right]$ there is $x$ such that $\sigma(x, t) \notin \mathcal{K}_{x}(t)$. Below we will focus on the time interval $\left[t_{1}, t_{2}\right]$.

We define function

$$
f(t)=\sup _{x \in M} d\left(\sigma(x, t), \mathcal{K}_{x}(t)\right) \text { for } t \in\left[t_{1}, t_{2}\right]
$$


where $d$ is the distance on $V_{x}$ defined by the metric $h$. It is clear that $f\left(t_{1}\right)=0$ and $f(t)>0$ for $t>t_{1}$.

Since the avoidance space-time track $\mathcal{A T}$ is closed and $\sigma(x, t) \notin \mathcal{A T}$ for all $x \in M$ and $t \in\left[t_{1}, t_{2}\right]$, there is an $\varepsilon>0$ such that

$$
\inf _{x \in M, t \in\left[t_{1}, t_{2}\right]} d\left(\sigma(x, t), \mathcal{A}_{x}(t)\right) \geq 3 \varepsilon .
$$

By Proposition 13 we have $(F(x, v, t), 1) \in C_{(v, t)}\left(\mathcal{T}_{x}\right)$ for all $(v, t) \in$ $\left(\partial \mathcal{T}_{x}\right) \backslash\left(\mathcal{A} \mathcal{T}_{x}\right)$, however we have used the property $(F(x, v, t), 1) \in C_{(v, t)} \mathcal{T}_{x}$ for all $(v, t) \in \partial \mathcal{T}_{x}$ in the proof of Lemma 12, we need to modify the proof of Lemma 12 to show that $f(t)$ is left lower semi-continuous and right-continuous. We adopt the notations used in the proof of Lemma 12 and replace $\widehat{\sigma}(x, t)$ by $\sigma(x, t)$. When $t_{a}=t_{1},\left(v_{\infty}, t_{1}\right)=\left(\sigma\left(x, t_{1}\right), t_{1}\right) \in$ $\left(\partial \mathcal{T}_{x_{\infty}}\right) \backslash\left(\mathcal{A} \mathcal{T}_{x_{\infty}}\right), f(t)$ is right-continuous at $t_{1}$ by the same proof. Hence there is $t_{3} \in\left(t_{1}, t_{2}\right)$ such that $f(t) \leq \varepsilon$ for all $t \in\left(t_{1}, t_{3}\right)$. For any $t_{a} \in\left(t_{1}, t_{3}\right)$

$$
d\left(v_{\infty}, \mathcal{A}\left(t_{a}\right)\right) \geq d\left(\sigma\left(x_{\infty}, t_{a}\right), \mathcal{A}\left(t_{a}\right)\right)-d\left(v_{\infty}, \sigma\left(x_{\infty}, t_{a}\right)\right) \geq 2 \varepsilon,
$$

so $\left(v_{\infty}, t_{a}\right) \in\left(\partial \mathcal{T}_{x_{\infty}}\right) \backslash\left(\mathcal{A} \mathcal{T}_{x_{\infty}}\right)$ for all $t_{a} \in\left(t_{1}, t_{3}\right)$ and $f(t)$ is left lower semicontinuous and right-continuous on $\left[t_{1}, t_{3}\right]$.

We will prove that there is a constant $C<+\infty$ such that $\frac{d^{+} f(t)}{d t} \leq C \cdot f(t)$ for all $t \in\left(t_{1}, t_{3}\right)$, then by Lemma 7 we get $f(t)=0$ for all $t \in\left[t_{1}, t_{3}\right]$, which is the required contradiction.

For any $t \in\left(t_{1}, t_{3}\right)$ since $f(t)=\sup _{x \in M} d\left(\sigma(x, t), \mathcal{K}_{x}(t)\right)<\varepsilon$, all the points in $\mathcal{T}$ we choose in the proof of Theorem 3 are at least $2 \varepsilon$ away from $\mathcal{A} \mathcal{T}$, so we can repeat the proof of Theorem 3 to conclude that $\frac{d^{+} f(t)}{d t} \leq C \cdot f(t)$ for all $t \in\left(t_{1}, t_{3}\right)$. Hence Theorem 4 is proved.

Acknowledgement. P.L. would like to thank Pengfei Guan for helpful discussions and Gang Tian for his constant support and encouragement.

\section{References}

[CK] B. Chow and D. Knopf, The Ricci Flow, Volume I: An Introduction, Mathematical Surveys and Monographs series, American Mathematical Society, Providence, 2004.

[H1] R.S. Hamilton, Three-manifolds with positive Ricci curvature, J. Differential Geom., 17 (1982), 255-306, MR 0664497, Zbl 0504.53034.

[H2] _ Four-manifolds with positive curvature operator, J. Differential Geom., 24 (1986), 153-179, MR 0862046, Zbl 0628.53042.

[H3] _ The Harnack estimate for the Ricci flow, J. Differential Geom., 37 (1993), 225-243, MR 1198607, Zbl 0804.53023.

[H4] - The formation of singularities in the Ricci flow, Surveys in Differential Geometry, 2, International Press, 1995, 7-136, MR 1375255, Zbl 0867.53030.

[H5] _ Four-manifolds with positive isotropic curvature, Comm. Anal. Geom., 5 (1997), 1-92, MR 1456308, Zbl 0892.53018. 
[H6] Nonsingular solutions of the Ricci Flow on three-manifolds, Comm. Anal. Geom., 7 (1999), 695-729, MR 1714939, Zbl 0939.53024.

[PW] M.H. Protter and H.F. Weinberger, Maximum Principles in Differential Equations, Springer-Verlag, 1984, MR 0762825, Zbl 0549.35002.

[S] J. Smoller, Shock Waves and Reaction-Diffusion Equations, Second edition, Grundlehren der Mathematischen Wissenschaften, 258, Springer-Verlag, 1994, MR 1301779, Zbl 0807.35002.

Received May 29, 2002 and revised August 5, 2003. The first author was supported by NSF Grant DMS-0203926.

Department of Mathematics

University of California, San Diego

LA JOLLA, CA 92093

E-mail address: benchow@math.ucsd.edu

Department of Mathematics and Statistics

MCMASTER UNIVERSITY

HAMILTON, ONTARIO

Canada, L8S 4K1

Department of Mathematics

UNIVERSITY OF OREGON

Eugene, OR 97403

E-mail address: penglu@darkwing.uoregon.edu 